\title{
Úlcera lingual como manifestação de tuberculose sistêmica - relato de caso
}

\section{Tongue ulcer as manifestation of systemic tuberculosis - case report}

\author{
Emerson Lucas da Silva Coimbra ${ }^{1}$. Bruno Augusto Benevenuto de Andrade ${ }^{2}$. Monica Lage da Rocha ${ }^{3}$. \\ 1 Universidade Salgado de Oliveira (UNIVERSO), Niterói, Rio de Janeiro, Brasil. 2 Universidade Estadual de Campinas \\ (UNICAMP), Piracicaba, São Paulo, Brasil. 3 Universidade Federal Fluminense (UFF), Niterói, Rio de Janeiro, Brasil.
}

\section{RESUMO}

A tuberculose é uma doença infecciosa crônica causada pelo mycobacterium tuberculosis. Lesões orais da tuberculose, embora incomuns, podem ser observadas e se caracterizam como úlceras crônicas e indolores. O objetivo desse trabalho é relatar um caso clínico de um paciente masculino, apresentando tuberculose em boca. Paciente procurou o serviço de estomatologia do Hospital Municipal Rocha Maia, RJ, Brasil, com queixa principal de dificuldade ao se alimentar e rouquidão, além de tosse e perda de apetite. Foi solicitada tomografia de tórax evidenciando no pulmão áreas de opacidade em vidro fosco e lesão nodular. Ao exame físico intrabucal observa-se lesão ulcerada extensa em ventre e borda lateral de língua e assoalho de boca, dolorosa e de borda endurecida. No exame de baciloscopia nota-se positividade para o mycobacterium tuberculosis. Diante de todos os achados foi realizada a biópsia incisional, e o exame histopatológico revelou diagnóstico de tuberculose. O tratamento proposto foi $2 \mathrm{RHZE}$ e $4 \mathrm{RHb}$ na fase de manutenção sendo 3 comprimidos ao dia. O presente relato mostra a importância da realização de uma boa anamnese e exame clínico minucioso pelo cirurgião dentista considerando que a lesão oral da tuberculose é diagnóstico diferencial para o carcinoma de células escamosas e outras doenças infecciosas.

Palavras-chave: Tuberculose. Manifestações bucais. Diagnóstico. Terapêutica.

\section{ABSTRACT}

Tuberculosis is a chronic infectious disease caused by mycobacterium tuberculosis. Oral lesions of tuberculosis, although uncommon, can be observed and are characterized as chronic and painless ulcers. The aim of this paper is to report a case report of a male patient with tuberculosis in the mouth. The patient sought the stomatology service of the Hospital Municipal Rocha Maia, RJ, Brazil, with the main complaint of difficulty eating and hoarseness, as well as cough and loss of appetite. Thorax tomography was requested, showing areas of ground-glass opacity and nodular lesion in the lung. In the intrabucal physical examination extensive ulcerated lesion in the belly and lateral border of the tongue and floor of the mouth, painful and hardened edge is observed. On smear examination, positivity for mycobacterium tuberculosis is noted. In the face of all findings, an incisional biopsy was performed, and histopathological examination revealed a diagnosis of tuberculosis. The proposed treatment was 2RHZE and 4RHb in the maintenance phase being 3 tablets a day. This report shows the importance of a good medical history and thorough clinical examination by the dentist considering that oral tuberculosis injury is a differential diagnosis for squamous cell carcinoma and other infectious diseases.

Keywords: Tuberculose. Manifestações bucais. Diagnóstico. Terapêutica.

Autor correspondente: Emerson Lucas da Silva Coimbra, Rua Princesa Margareth, número 20, Cabuçu, Nova Iguaçu, Rio de Janeiro. CEP: 26291602. Telefone: +55 21 97579-4789. E-mail: emersoncoimbra@outlook.com

Conflito de interesses: Não há qualquer conflito de interesses por parte de qualquer um dos autores.

Recebido em: 23 Jul 2019; Revisado em: 16 Dez 2020; Aceito em: 09 Fev 2021. 


\section{INTRODUÇÃO}

A tuberculose (TB) existe há muitos anos e continua sendo um problema de saúde global. É definida como uma doença infecciosa granulomatosa crônica causada pela bactéria mycobacterium tuberculosis sendo uma das principais causas de morte nos países em desenvolvimento. De acordo com o relatório da Organização Mundial da Saúde em 2018, é a nona causa de morte em todo o mundo. ${ }^{1}$

$\mathrm{Na}$ maioria das vezes afeta os pulmões, embora alguns pacientes apresentem a doença em outras partes do corpo. A TB extrapulmonar é responsável por $25 \%$ dos casos, com 10 a $35 \%$ detectados na região da cabeça e pescoço. ${ }^{2}$

Embora, as manifestações orais da tuberculose sejam incomuns, são observadas em $0,5 \%$ a $5 \%$ dos pacientes. ${ }^{3,4,5}$ A TB oral pode ser classificada como primária ou secundária. Na forma primária, a cavidade oral é o local inicial de infecção por inoculação direta de bactérias, enquanto a TB secundária surge subsequente à disseminação da infecção tuberculosa de outro local via linfática e hematogênica. A forma secundária é mais comumente observada que a primária. ${ }^{6}$

Geralmente, a manifestação bucal é observada como uma lesão ulcerada e indolor. O local mais comumente afetado é a língua, seguida por palato, lábios, mucosa jugal, gengiva, acompanhado de linfadenopatia cervical persistente. ${ }^{3,6}$ Embora a maioria dos casos de TB oral se apresente com uma lesão ulcerativa crônica da mucosa, ela também pode se manifestar semelhante a uma doença granulomatosa, osteomielite ou neoplasia da glândula salivar. ${ }^{3}$

Assim, o presente estudo tem como objetivo relatar um caso clínico de tuberculose secundária em cavidade oral, assim como os aspectos clínicos, histopatológicos e conduta terapêutica.

\section{CASO CLÍNICO}

Paciente melanoderma, do gênero masculino, 35 anos, morador da comunidade da Rocinha no Rio de Janeiro - RJ, Brasil, foi encaminhado pela clínica Família ao serviço de estomatologia do Hospital Municipal Rocha Maia, RJ, Brasil, com queixa principal de úlcera na língua, com meses de evolução.

A história médica relevou que o paciente apresentava dificuldade de se alimentar, apresentando rouquidão e tosse seca e persistente há três meses, além de perda de apetite, emagrecimento e febre vespertina. O mesmo relatou ser usuário de maconha e cocaína há 20 anos.

Foi solicitada uma tomografia de tórax evidenciando no pulmão áreas de infiltrado nos ápices dos lobos superiores e segmentos superiores dos lobos inferiores, e opacidade em vidro fosco e lesão nodular.

Ao exame extraoral, foi detectado um nódulo linfático submandibular aumentado do lado esquerdo e de consistência firme e móvel.
Ao exame físico intrabucal observou-se uma lesão ulcerada extensa, com halo eritematoso, medindo aproximadamente $5 \mathrm{~cm}$ em seu maior diâmetro em assoalho de boca, ventre e borda lateral esquerda de língua, dolorosa e de bordas irregulares, elevadas e endurecidas. (Figura 1).

Figura 1. Fotografia da região de borda lateral e ventre de língua e assoalho da boca mostrando lesão ulcerada.

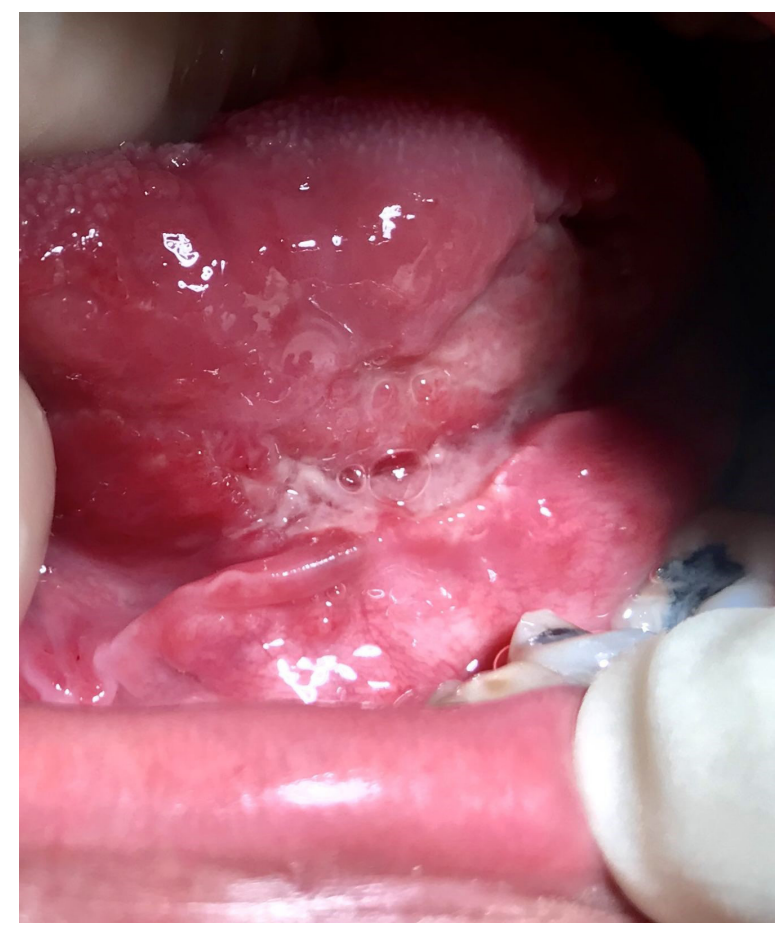

Diante dos achados clínicos, foi sugerido algumas hipóteses diagnósticas como carcinoma de células escamosas, tuberculose secundária, paracoccidioidomicose, linfoma ou reação a drogas.

Foram realizados testes rápidos para Sífilis, HIV e Hepatite, sendo todos negativos e no exame de baciloscopia notou-se positividade para o mycobacterium tuberculosis.

Uma biópsia incisional da úlcera foi realizada sob anestesia local (Figura 2). O exame histopatológico da amostra mostrou presença de inflamação crônica granulomatosa caracterizada pela formação de múltiplos granulomas formados por macrófagos de aspecto epitelioide além de células gigantes multinucleadas do tipo langhans e necrose caseosa (Figura 3). A coloração de Ziehl-Neelsen (BAAR) assim como PAS e GROCOTT foram negativas (Figura 4 e 5). Associando os achados clínicos, laboratoriais e microscópicos, foi confirmado o diagnóstico de tuberculose extrapulmonar secundária em cavidade oral. 
Figura 2. Fragmento de tecido da biópsia incisional em região de borda lateral e assoalho da boca.

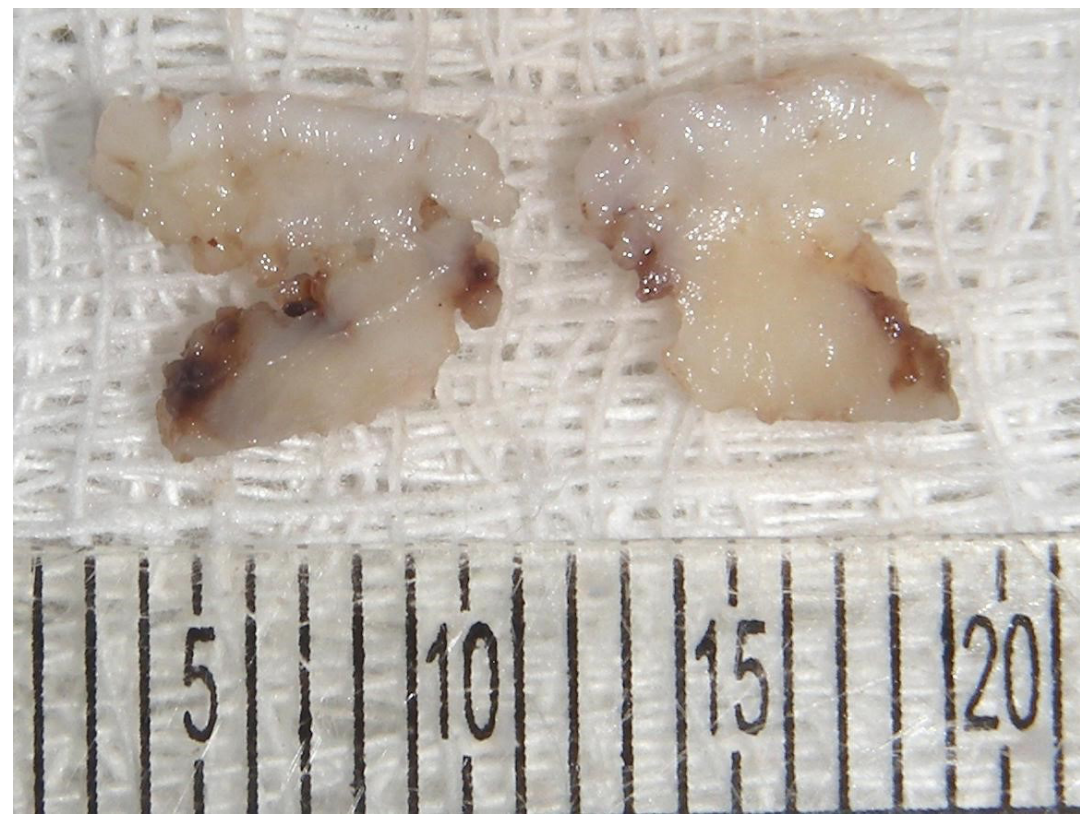

Figura 3. Imagens histológicas do caso de tuberculose oral.
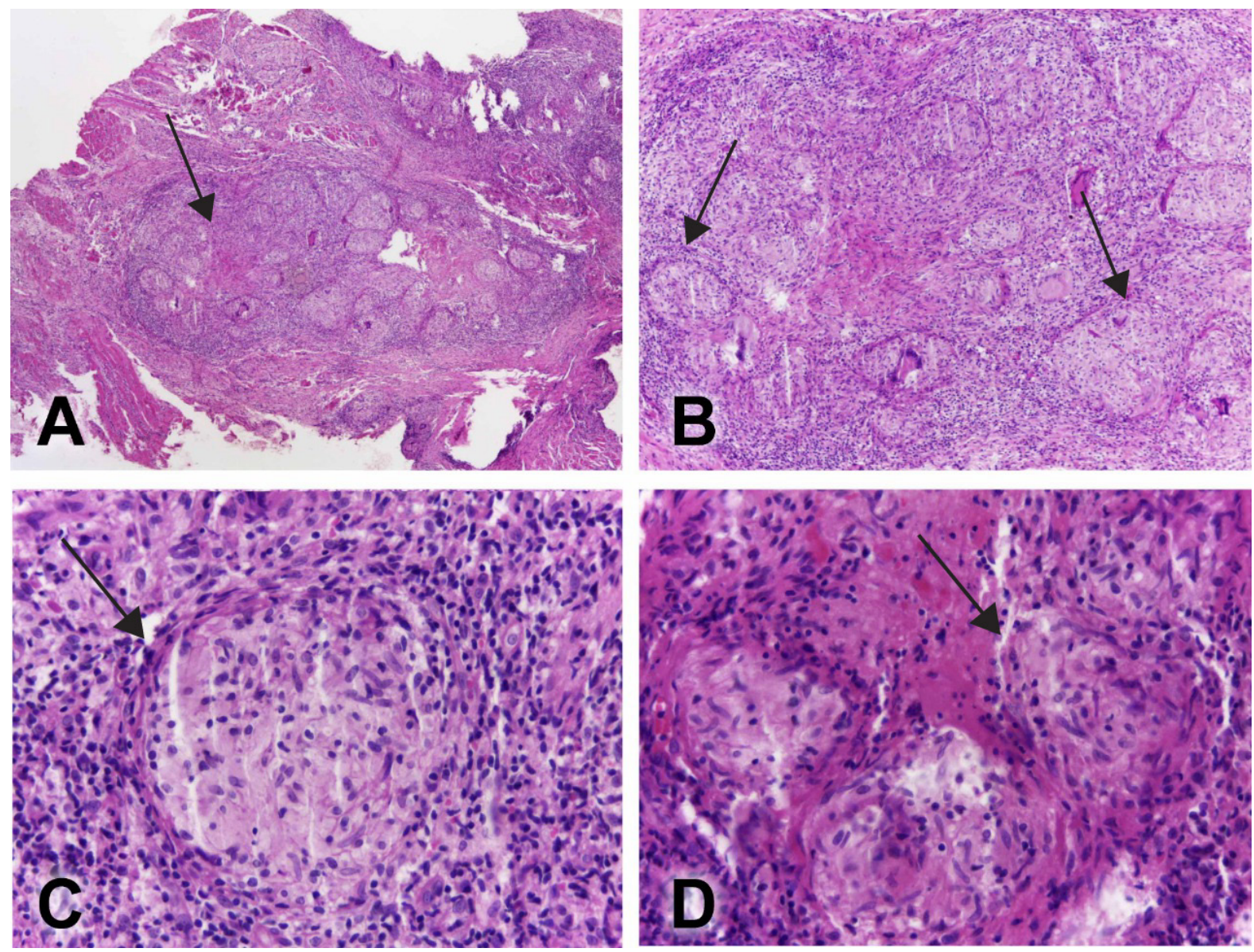

(A) Corte de tecido corado com hematoxilina e eosina mostrando no tecido conjuntivo presença de inflamação crônica granulomatosa (ampliação original, 40x). (B) Presença de múltiplos granulomas compostos por macrófagos epitelioides e linfócitos nas áreas circundantes, além de algumas células gigantes multinucleadas de langerhans no centro (ampliação original, 100x). (C-D) Visão em maior aumento onde observa-se com melhor detalhe os granulomas (ampliação original, 400x). 
Figura 4. A coloração de Ziehl-Neelsen (BAAR) para bacilos ácidos resistentes utilizando ácido sulfúrico a $20 \%$ mostrou negatividade.

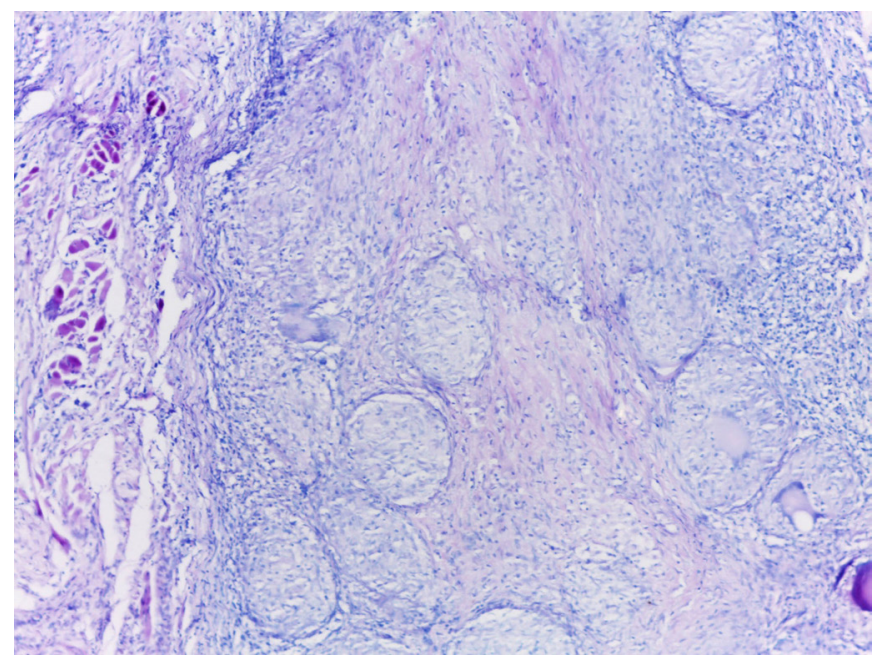

Figura 5. A coloração de GROCOTT-GOMORI (A) e PAS (B) mostrou-se negativa descartando a possibilidade de doenças fúngicas.
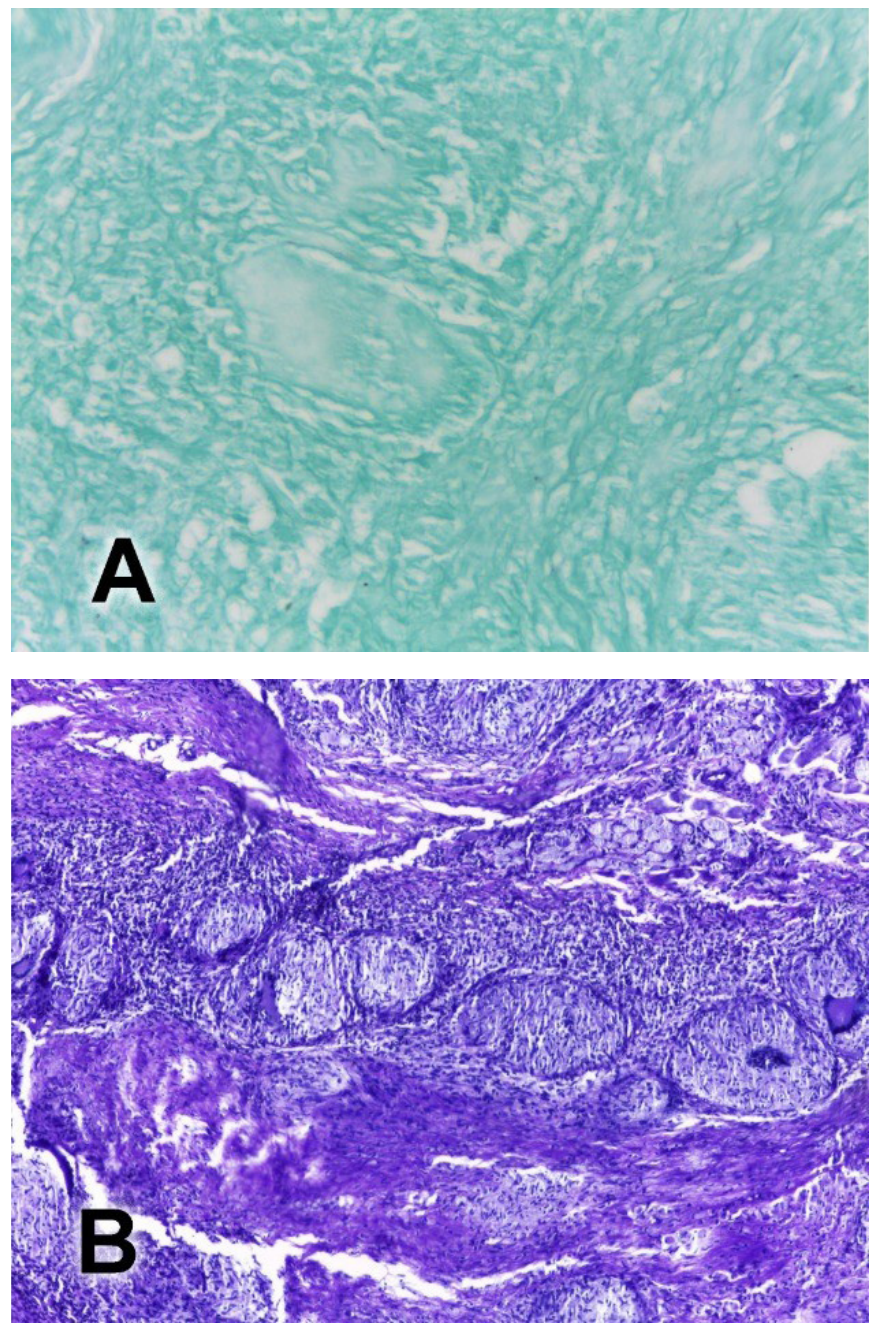

Após o diagnóstico, o paciente retornou para clínica da Família da Rocinha, para o tratamento proposto pelo Ministério da Saúde. O esquema Intensivo foi realizado com (2RHZEa), uma combinação de rifampicina (R), isoniazida $(\mathrm{H})$, pirazinamida $(\mathrm{Z})$ e etambutol $(\mathrm{E})$. Sendo as doses de RHZE 150/75/400/275mg em comprimido em dose fixa combinada, sendo 3 comprimidos por dia, durante 2 meses. A fase de manutenção (4RHb) foi realizado o esquema RH: combinação de rifampicina $(\mathrm{R})$ e isoniazida $(\mathrm{H})$, Sendo as doses de 150/75mg em comprimido em dose fixa combinada, sendo 3 comprimidos ao dia, durante 4 meses.

Após o esquema intensivo foi observado a regressão de parte da lesão. O paciente permanece em acompanhamento clínico (Figura 6).

Figura 6. Fotografia da região de borda lateral e ventre de língua e assoalho evidenciando processso de cicatrização após início da terapia medicamentosa.

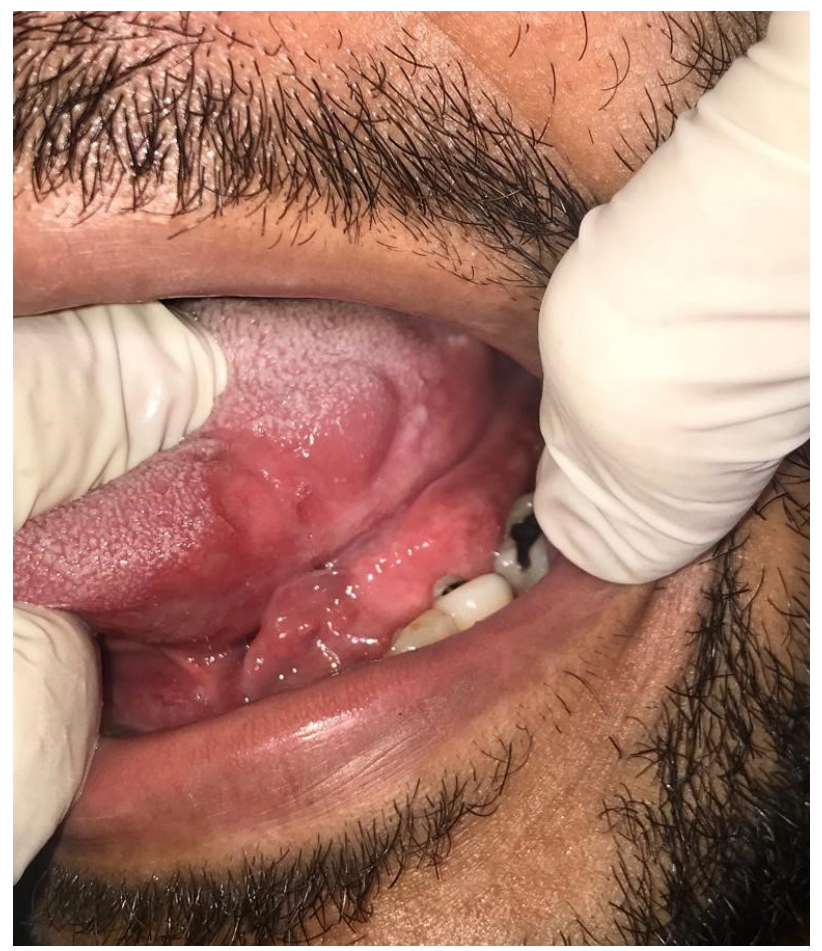

\section{DISCUSSÃO}

A tuberculose é uma doença infecciosa granulomatosa crônica causada pelo mycobacterium tuberculosis e continua sendo uma das principais causas de morbidade e mortalidade nos países em desenvolvimento, especialmente no Brasil onde em 2018, foram diagnosticados 72.788 casos novos, o que corresponde a um coeficiente de incidência de 34,8 casos/100 mil habitantes segundo o relatório global de tuberculose da Organização Mundial da Saúde de 2018. ${ }^{1,7}$

Embora a tuberculose afete principalmente os pulmões, pode também apresentar-se em outras partes do corpo, incluindo a cavidade oral. Manifestações orais da TB são uma ocorrência 
rara, e ocorrem em aproximadamente $0,5-5 \%$ de todos os casos de infecções pela TB. ${ }^{6,8,9}$

A manifestação oral da tuberculose pode ser classificada como primária ou secundária. A TB primária não apresenta comprometimento pulmonar, e é extremamente rara, sendo mais comumente encontrada em crianças e adolescentes. Clinicamente apresenta-se, principalmente como uma úlcera indolor, comumente envolvendo a região de gengiva, prega mucovestibular e áreas de inflamação adjacente aos dentes ou locais de extração, e está associada com linfadenopatia regional. ${ }^{10,11}$

A TB oral secundária é mais comum, sendo frequentemente associada à doença pulmonar, principalmente em pacientes de meia-idade e idosos. O dorso da língua é o local mais acometido seguido pelo palato, mucosa jugal, lábios, úvula e amígdalas. Mandíbula, maxila, seios paranasais e articulação temporomandibular podem ser afetadas, apresentando osteomielite tuberculosa. ${ }^{10,11,12}$

A raridade da manifestação da tuberculose na boca está relacionada principalmente ao epitélio escamoso espesso e intacto da cavidade oral, que resiste à penetração direta pelos bacilos. Esta resistência pode também ser atribuída à ação da saliva, pH local, e anticorpos. Na TB primária, a entrada do organismo pode ser através de uma ruptura na membrana mucosa causada por trauma mecânico, irritação crônica e doenças periodontais pré-existentes que podem favorecer a inoculação direta do microorganismo. A TB secundária ocorre devido à disseminação hematogênica ou linfática e extensões de estruturas vizinhas infectadas pelo mycobacterium tuberculosis, que gera uma auto-inoculação resultante principalmente da expectoração infectada. ${ }^{12,13}$

As lesões orais podem apresentar uma variedade de manifestações clínicas. As úlceras apresentam um aspecto crateriforme com halo eritematoso, podendo ser única ou múltiplas, sintomáticas ou não, com bordas irregulares e geralmente estão localizadas na parte posterior e na superfície ventral da língua. Quando TB ocorre na forma nodular, o curso clínico é mais persistente e doloroso. Nestes casos há linfadenopatia cervical indolor que, posteriormente, podem formar abscessos..$^{12,13}$

O diagnóstico clínico dessas lesões geralmente se torna difícil, já que outras lesões como úlcera traumática, carcinoma de células escamosas, sífilis, histoplasmose e blastomicose são diagnósticos diferenciais. ${ }^{10} \mathrm{O}$ diagnóstico diferencial histopatológico inclui outras condições granulomatosas, como a sarcoidose, doença de Crohn, micoses profundas, doença da arranhadura do gato, reações de corpo estranho e sífilis terciária. A história clínica e exames complementares são importantes para o diagnóstico, incluindo testes de laboratório e imagens radiográficas. ${ }^{9}$ A apresentação clínica do nosso caso sugeriu diagnóstico inicial de carcinoma de células escamosas.

O diagnóstico confirmatório da tuberculose pode ser realizado por diversos métodos, sendo que o mais utilizado é a baciloscopia direta onde é realizado a pesquisa do bacilo álcool-ácido resistente - BAAR, pelo método de ZiehlNielsen. Outros testes podem ser realizados como a cultura de escarro e evidência radiográfica. Devem ser seguidos passos obrigatórios para descartar TB sistêmica como uma radiografia de tórax e um teste de tuberculina (Montoux). ${ }^{10}$

O exame histopatológico revela uma inflamação granulomatosa com células gigantes de Langhans e necrose caseosa, além da identificação dos bacilos usando corantes especiais. O estudo histopatológico é necessário para excluir outras infecções e confirmar diagnóstico definitivo de TB. ${ }^{6}$

Após a confirmação do diagnóstico de tuberculose, o paciente deve ser tratado com fármacos anti-tuberculose em duas fases, sendo que na fase intensiva do regime envolve combinação de quatro tipos de medicamentos isoniazida, rifampicina, pirazinamida e etambutol em 2 meses administrados diariamente nos dois primeiros meses, seguidos pela fase de manutenção de quatro meses com apenas três medicamentos que incluiu Isoniazida, Rifampicina e etambutol é essencial para a completa erradicação das lesões tuberculares. Embora o tratamento de 6 meses pode ser suficiente para muitos pacientes, cada doente deve ser avaliado individualmente. ${ }^{14,15}$

\section{CONCLUSÕES}

Apesar de ser uma manifestação rara, lesões orais de tuberculose devem ser incluídas no diagnóstico diferencial, independentemente da existência de sinais e sintomas pulmonares, e se o paciente vive ou não em uma região endêmica de TB. O diagnóstico precoce é essencial para o estabelecimento de um tratamento adequado, visto que o diagnóstico tardio aumentará a morbidade e a mortalidade dos pacientes, como também o risco de transmissão da doença. Por isso, torna-se importante o conhecimento pelo cirurgião-dentista para incluir a tuberculose no diagnóstico diferencial de lesões orais ulceradas endurecidas e de longa duração como suspeitas, para evitar o atraso no diagnóstico e tratamento.

\section{REFERÊNCIAS}

1. World Health Organization. Global tuberculosis report 2018. Geneva: World Health Organization; 2018 [acesso em: 25 Set 2019]. Disponível em: http://www. who.int/iris/handle/10665/274453

2. Souza BC, Lemmos VM, Munerato MC. Oral manifestation of tuberculosis: a case-report. Braz J Infect Dis. 2013;20(2):210-13.

3. Santiago RA, Gueiros LA, Porter SR, Gomes VB, Ferrer I, Leão
JC. Prevalence of oral lesions in Brazilian patients with tuberculosis. Indian J Dent Res. 2013;24(2):245-8.

4. Kakisi OK, Kechagia AS, Kakisis IK, Rafailidis PI, Falagas ME. Tuberculosis of the oral cavity: a systematic review. Eur J Oral Sci. 2010;118(2):103-9.

5. Vaid S, Lee YY, Rawat SA, Luthra D, Shah A, Ahuja T. Tuberculosis 
in the head and neck: a forgotten differential diagnosis. Clin Radiol. 2010;65(1):73-81.

6. Gupta G, Khattak BP, Agrawal V. Primary gingival tuberculosis: a rare clinical entity. Contemp Clinical Dent. 2011;2(1):31-3.

7. Brasil. Ministério da Saúde. Secretaria de Vigilância em Saúde. Brasil Livre da Tuberculose: evolução dos cenários epidemiológicos e operacionais da doença. Boletim epidemiológico. 2019;50(9):1-18.

8. Wu Y, Chang Y, Sun A, Chiang C. Oral tuberculosis. J Form Med Ass. 2017;116(1):64-5.

9. Miziara ID. Tuberculosis affecting the oral cavity in Brazilian HIV-infected patients. Oral Surg Oral Med Oral Pathol Oral Radiol Endod. 2005;100(2):179-82

10. Jain P, Jain I. Oral manifestations of tuberculosis: step towards early diagnosis. J Clin Diagn Res. 2014;8(12):18-21.
11. Mignogna MD, Muzio LL, Favia G, Ruoppo E, Sammartíno G, Zarrelli C, et al. Oral tuberculosis: a clinical evaluation of 42 cases. Oral Dis. 2000;6(1):25-30.

12. Mahajan S, Srikant N, George T. Atypical presentation of oral tuberculosis ulcer. N Y State Dent J. 2007;73(6)48-50.

13. Dinnes J, Deeks J, Kunt H, Gibson A, Cummins E, Waugh N, et al. A systematic review of rapid diagnostic tests for the detection of tuberculosis infection. Health Technol Assess. 2007;11(3):191-6.

14. Ramírez-Lapausa M, Menéndez-Saldaña A, Noguerado-Asensio A. Extrapulmonary tuberculosis: an overview. Rev Esp Sanid Penit. 2015;17(1):3-11.

15. Jann SM, Khan FY, Bhat MA, Behal R. Primary tuberculous gingival enlargement - a rare clinical entity: case report and brief review of the literature. J Indian Soc Periodontol. 2014;18(5):632-6.

\section{Como citar:}

Coimbra EL, Andrade BA, Rocha ML. Úlcera lingual como manifestação de tuberculose sistêmica - relato de caso. Rev Med UFC. 2021;61(1):1-6. 